Trauma Berufskrankh $2005 \cdot 7$ [Suppl 2]: S327-S331 DOI 10.1007/s10039-004-0961-y

Online publiziert: 27. November 2004

(c) Springer Medizin Verlag 2004

M. Bierschneider · B. Boszczyk · H. Jaksche · BG-Unfallklinik, Murnau

\title{
Risiken der Vertebro- und Kyphoplastie
}

\section{Risiken}

\section{Präoperativ}

Bei der Indikationsstellung zu einem minimalinvasiven Augmentationsverfahren sollte sich der Behandler folgende Fragen stellen:

- Ist es das richtige Verfahren?

- Ist es der richtige Patient?

- Bin ich der richtige Operateur?

- Ist es der richtige Wirbel?

- Ist es das richtige Material?

\section{Richtiges Verfahren}

Grundsätzlich sind weder die Vertebroplastie noch die Kyphoplastie geeignet, die etablierten Verfahren der Wirbelsäulenchirurgie zu ersetzen. Sie können für ausgewählte Patienten eine Alternative so- wohl zur konservativen als auch zur offenen operativen Therapie darstellen.

Die Indikationsstellung für die Vertebro- bzw. Kyphoplastie beschränkt sich auf Patienten mit Frakturen des Typs A1.1, A1.2 sowie, in ausgewählten Fällen, A3.1. Grundsätzlich ist bei jeder Art von spinaler oder foraminaler Stenose mit entsprechender klinischer Ausfallssymptomatik (Claudicatio spinalis, radikuläre Ausfälle) große Vorsicht geboten, da sich diese Probleme durch eine Zementeinspritzung nicht beheben lassen ( $\bullet$ Abb. 1).

\section{Richtiger Patient}

Ein minimalinvasives Verfahren macht einen inoperablen Patienten nicht zum operablen.

Der Patient muss narkosefähig sein oder, wenn man den Eingriff in Lokalanästhesie durchführen will, zumindest in der

Bei der Vertebroplastie wird nach Einbringen der Injektionskanüle in den Wirbelkörper dünnflüssiger Knochenzement unter hohem Druck injiziert.

Bei der Kyphoplastie wird über ein spezielles Kanülensystem ein mit Kontrastmittel gefüllter, expandierbarer Ballon in den Wirbelkörper eingebracht. Durch Aufblasen desselben wird ein Hohlraum geschaffen. Nach Entfernen des Ballons kann dieser mit zähflüssigem Knochenzement unter geringem Druck und somit mit geringerer Gefahr des Zementaustritts gefüllt werden.

Prinzipiell beinhalten beide Verfahren in allen Entscheidungs- und Handlungsebenen, d. h. prä-, intra- und postoperativ, mögliche Risiken.
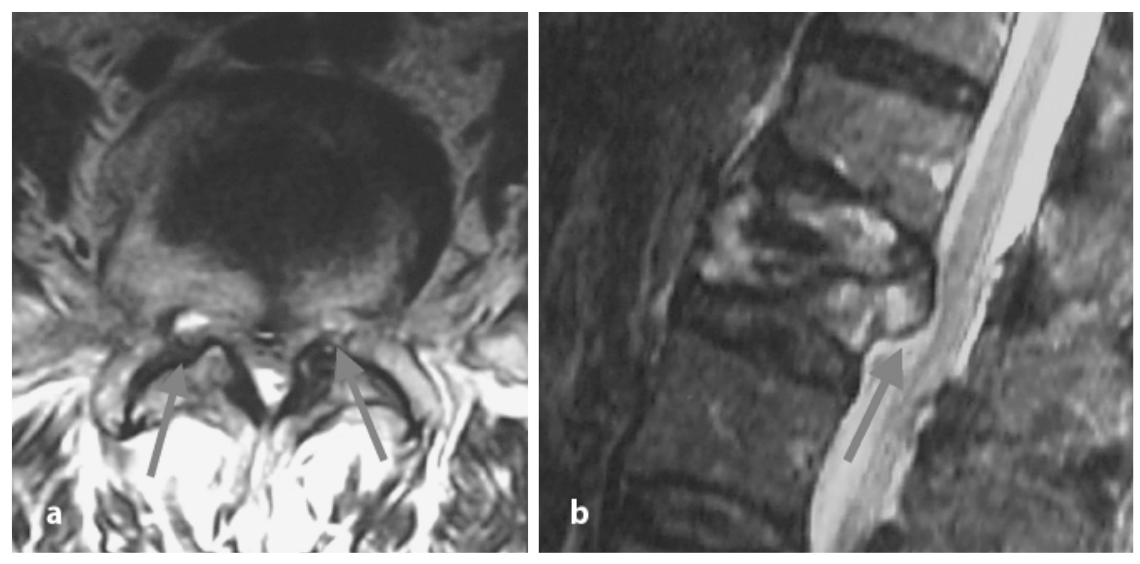

Abb. $1 \Delta$ Grenzen der perkutanen Technik, a begleitende Recessusstenose, b Hinterkantenfragment mit konsekutiver Spinalstenose 


\section{Ventrale Instrumentation BWS/LWS}
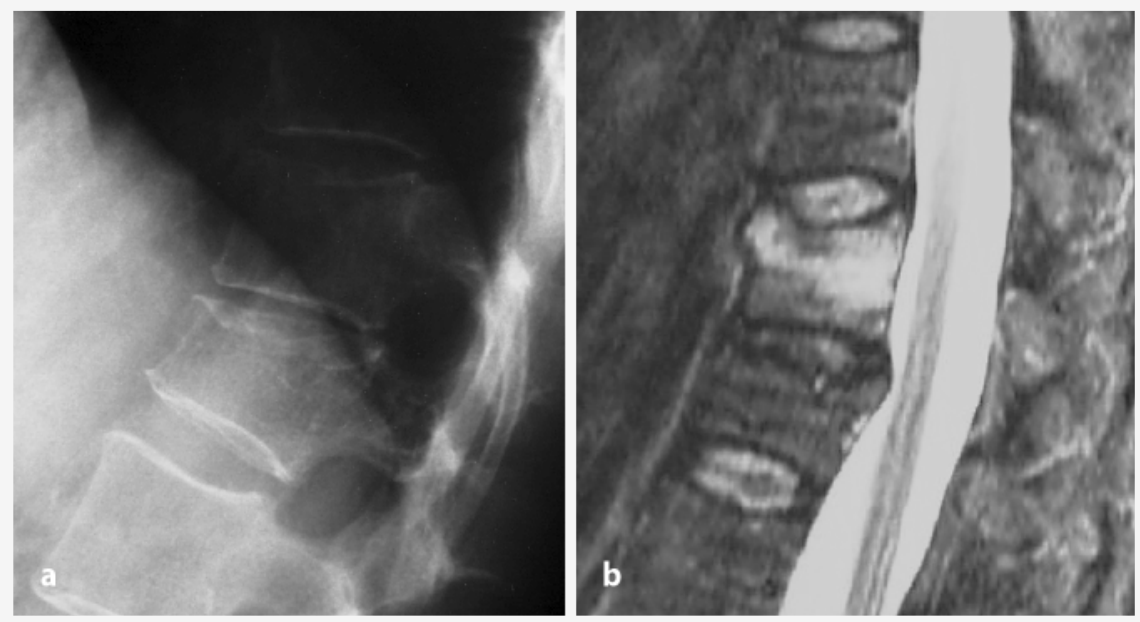

Abb. $2 \triangle$ Röntgen- vs. MRT-Befund, a Nativröntgen: LWK1 erscheint deformiert, b MRT (STIR-Sequenz): Wirbel über LWK1 frisch frakturiert
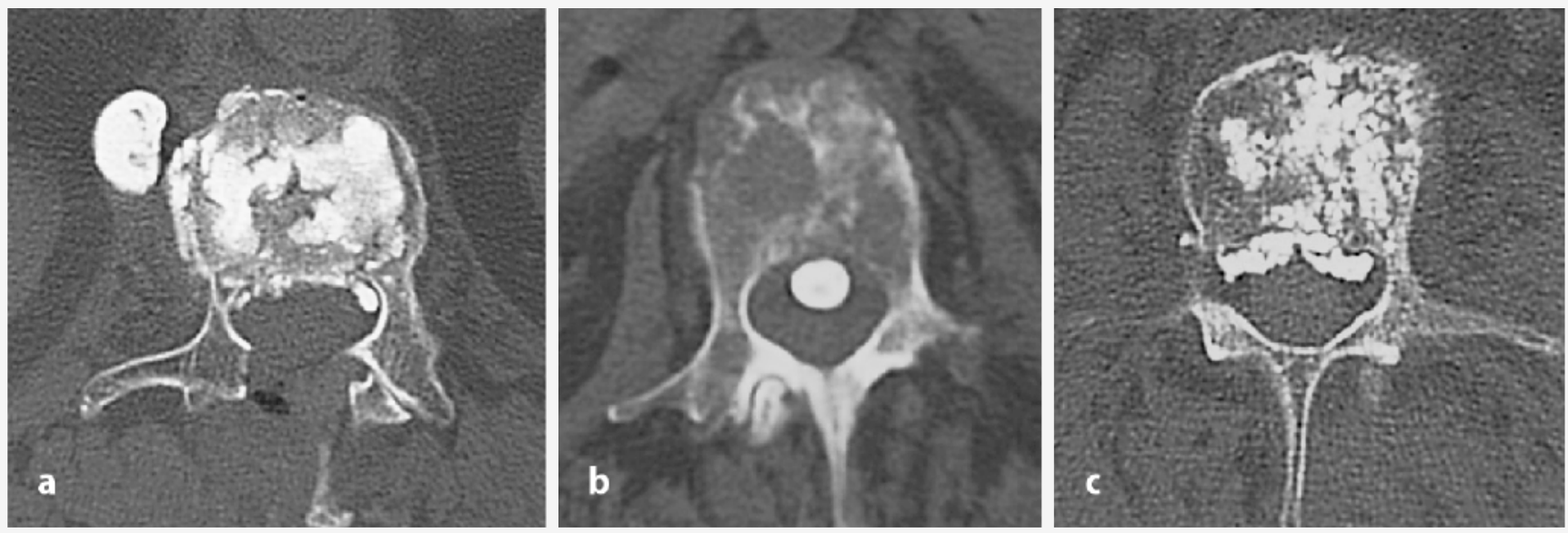

Abb. $3 \triangle$ Vertebroplastie, Zementaustritt, a paravertebral, b intraspinal, c mit Ausguss epiduraler Venen

Lage sein, für die Dauer des Eingriffs auf dem Bauch zu liegen. Durch massive Adipositas des Patienten können sowohl die Identifikation der anatomischen Landmarken, die zur Platzierung der Instrumente notwendig sind, als auch die Beobachtung des Knochenzements während der Injektion derartig erschwert sein, dass eine sichere Durchführung des Verfahrens nicht möglich ist. Dies gilt im Besonderen für den hochthorakalen und lumbosakralen Bereich, da hier durch benachbarte anatomische Strukturen (Schultern bzw. Becken) radiologische Überlagerungen auftreten können.

Des Weiteren müssen bei der Auswahl der Patienten die absoluten Kontraindikationen für beide Verfahren beachtet werden:

1. Asymptomatische Wirbelkörperfrakturen
2. Therapierefraktäre Koagulopathie oder hämorrhagische Diathese

3. Bakterielle Infektionen

\section{Richtiger Operateur}

Ausreichende operative Wirbelsäulenerfahrung ist zu fordern, des Weiteren sollte der Operateur den Nachweis über die Teilnahme an einem theoretisch-praktischen Kurs zum Erlernen der Vertebro- bzw. Kyphoplastie führen. Darüber hinaus sollte er in der Lage sein, Komplikationen, die einen Umstieg auf ein offenes operatives Verfahren erfordern, behandeln zu können.

\section{Richtiger Wirbel}

Es sollte große Sorgfalt auf die Identifizierung des für die Schmerzen verantwortlichen Wirbels gelegt werden. Dies ist bei den oft älteren Menschen mit langjähriger Rückenschmerzanamnese und entspre-

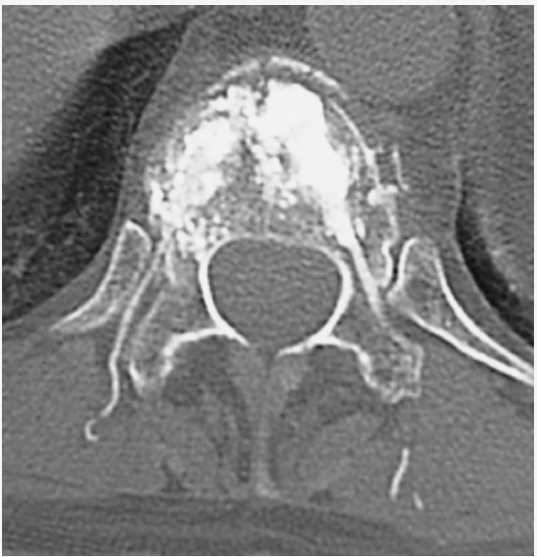

Abb. $4 \Delta$ Kyphoplastie, retrograder Zementaustritt in Muskulatur chenden degenerativen Veränderungen im Nativröntgenbild oft schwierig. Die klinischen Beschwerden des Patienten, das Nativröntgenbild sowie die Schnittbildgebung müssen hierbei übereinstimmen.

Im Zweifelsfalle hat sich hier die MRT mit Identifikation eines Knochenödems im betroffenen Wirbelkörper als äußerst hilfreich im klinischen Alltag erwiesen (• Abb. 2).

\section{Richtiges Material}

Eine Vielzahl von Knochenzementen befindet sich auf dem Markt. Für die Verwendung bei der Vertebro- bzw. Kyphoplastie müssen die Materialien definierten Ansprüchen genügen: Sie müssen

$$
\begin{aligned}
& \text { - gut injizierbar sein, } \\
& \text { - eine adäquate Röntgenkontrastge- } \\
& \text { bung haben, }
\end{aligned}
$$


- ein Verarbeitungsfenster von etwa 1012 min aufweisen und

- eine ausreichende Primärstabilität bieten.

In erster Linie werden Zemente auf Basis des Polymethylmethacrylats angewandt. Vergleichbare Ergebnisse wurden auch unter Verwendung von Kalziumphosphatzementen bei osteoporotischen Frakturen erzielt. Die Einsetzbarkeit derselben bei traumatische Frakturen des jüngeren Menschen wird derzeit untersucht.

\section{Intraoperativ}

Hier unterscheiden wir in erster Linie zugangs- und zementierungsbedingte Risiken.

\section{Zugangsbedingte Risiken}

Beim röntgengestützten Vorschieben der Kanüle in den betroffenen Wirbelkörper sind prinzipiell alle Strukturen und Organe in und um die Wirbelsäule herum in Gefahr, verletzt zu werden.

Bei medialem Abweichen der Kanüle besteht die Gefahr der Perforation in den Spinalkanal mit Verletzung des Rückenmarks bzw. der Cauda equina mit entsprechenden neurologischen Ausfallserscheinungen.

Bei einem lateralen Abweichen der Kanüle ist, in Abhängigkeit vom behandelten Wirbelsäulensegment, eine Perforation in die Lunge bzw. Abdominalorgane möglich. Bei einer Perforation nach anterior können große Gefäße verletzt werden.

Insgesamt wird die Verletzungsrate von benachbarten Strukturen auf den $\mathrm{Zu}$ gangsweg in den Wirbelkörper bei beiden Verfahren mit deutlich unter 1\% angegeben $[5,13]$. Die Dunkelziffer bei wenig geübten Anwendern scheint jedoch höher zu liegen.

Als Hauptursachen für die Verletzung benachbarter Strukturen werden in erster Linie eine schlechte Bildgebung sowie die mangelnde Erfahrung des Operateurs angesehen.

\section{Zementierungsbedingte Risiken}

Die häufigste Komplikation bei beiden Verfahren stellt der Zementaustritt in den Spinalkanal, das Neuroforamen, die umgebende Muskulatur sowie die Blutgefäße mit Verschleppung in die Lunge dar [4, $6,14,15,17,19,21,23,24,25]$. Die Zementaustrittsrate variiert deutlich zwischen beiden Verfahren, sie wird für die Vertebroplastie mit 10-70\% und für die Kyphoplastie mit $<10 \%$ angegeben $(\bullet$ Abb. 3,4$)$. Die ungleich höhere Rate an Zementaustritt für die Vertebroplastie ist in der unterschiedlichen Technik begründet: Dünnflüssiger Zement wird unter hohem Druck in den trabekulären Knochen injiziert. Bei der Kyphoplastie wird zähflüssiger Zement in die durch den Ballon geschaffene Höhle unter geringem Druck eingebracht.

Als Ursachen für einen Zementaustritt können neben einem zu dünnflüssigen Zement und einem zu hohen Injektionsdruck eine schlechte Bildgebung während der Injektionsphase und die Ungeduld des Operateurs während der Injektion genannt werden. Zur Vermeidung von Zementaustritten empfiehlt es sich daher, den Zement möglichst zäh, ohne großen Kraftaufwand, ohne zeitliche Hast bei guter Bildgebung zu injizieren. Bei der Vertebroplastie ist vor der Zementinjektion die Durchführung eines Venogramms mit Kontrastmittel zur Darstellung des venösen Abflusses zu empfehlen.

\section{Postoperativ}

Hier sind in erster Linie Infektion sowie Anschlussfraktur zu nennen.

\section{Infektion}

Die postoperative Infektion kann lokal im Zugangsbereich als Wundinfekt auftreten, sie kann sich in die paravertebralen Weichteile ausdehnen, in den Spinalkanal einbrechen, die Wirbelkörper oder die Bandscheibe betreffen.

Es gibt verschiedene Faktoren, die einen Patienten für das Auftreten einer postoperativen Infektion prädisponieren. An erster Stelle zu nennen sind Immunsuppression, Diabetes mellitus sowie Adipositas. Aber auch Sterilitätsfehler bei der Vorbereitung und Durchführung des Eingriffs sind ins Kalkül zu ziehen.

Um das Infektionsrisiko zu minimieren, sollten die Patienten entsprechend ihres persönlichen Risikoprofils unter Beachtung der Kontraindikationen selektiert werden, ggf. sollte eine perioperative An-
Trauma Berufskrankh 2005 - 7 [Suppl 2]:

S327-S331

DOI 10.1007/s10039-004-0961-y

c) Springer Medizin Verlag 2004

\author{
M. Bierschneider $\cdot$ B. Boszczyk \\ H. Jaksche
}

\section{Risiken der Vertebro- und Kyphoplastie}

\section{Zuammenfassung}

Vertebro- und Kyphoplastie haben sich als effektive minimalinvasive Verfahren zur Behandlung osteoporotischer Wirbelkörperfrakturen erwiesen. Unter Beachtung einiger Vorsichtsmaßnahmen sind die Risiken überschaubar. Die vorliegende Arbeit gibt einen Überblick über das Risikopotenzial beider Verfahren und zeigt Maßnahmen zur Fehlervermeidung auf.

\section{Schlüsselwörter}

Vertebroplastie · Kyphoplastie .

Komplikationen

\section{Risks involved in vertebroplasty and kyphoplasty}

\begin{abstract}
Vertebroplasty and kyphoplastie have proved to be effective minimally invasive methods in the treatment of osteoporotic vertebral fractures. If adequate precautions are taken the risks involved can be foreseen. This article gives a summary of potential risks and describes how complications can be avoided.
\end{abstract}

\section{Keywords}

Vertebroplasty · Kyphoplasty .

Complications 


\section{Ventrale Instrumentation BWS/LWS}
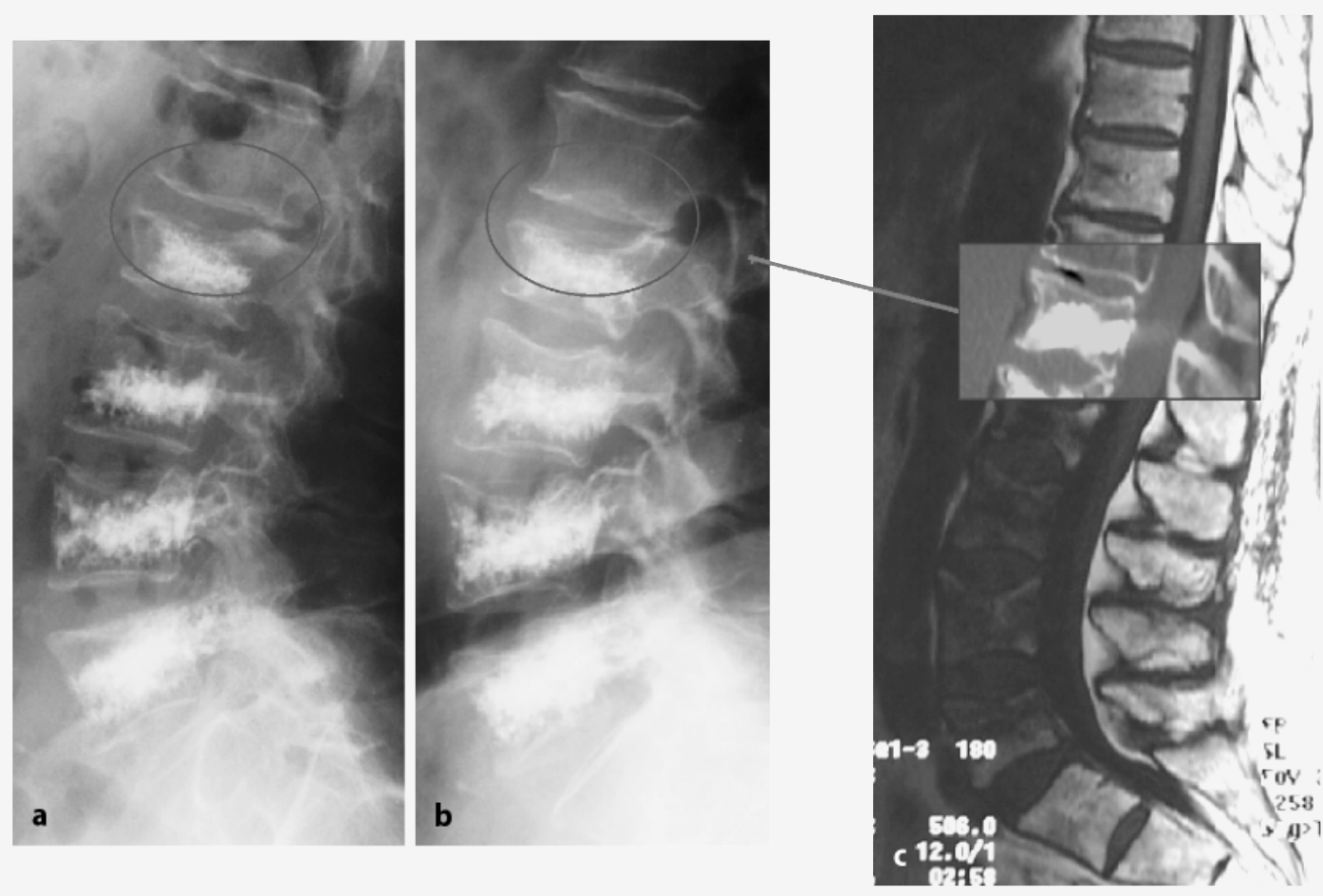

Abb. $5<$ a Vertebroplastie in 4 Höhen, $b$ Anschlussfraktur durch Deckplatteneinbruch, c Zustand nach Augmentation der Anschlussfraktur

tibiotikaprophylaxe durchgeführt werden. Des Weiteren sollte der Eingriff in einem adäquat ausgestatteten Eingriffsraum, mit den für operative Eingriffe üblichen hygienischen Kautelen, durchgeführt werden.

\section{Anschlussfraktur}

30-60\% neuer Frakturen sind angrenzend (• Abb. 5). Ein erhöhtes Risiko für das Auftreten einer Anschlussfraktur besteht bei multiplen vorbestehenden Frakturen sowie einer sekundären Osteoporose. Nach dem heutigen Kenntnisstand gehen wir davon aus, dass das Risiko einer Anschlussfraktur durch das Ausmaß der osteoporotischen Grunderkrankung bestimmt wird $[2,3,5,8,13,22,26]$. Abhilfe wird hier durch eine konsequente Behandlung der Grunderkrankung geschaffen.

\section{Fazit}

Minimalinvasive Technik heißt nicht minimales Risiko.

Die Risiken sind überschaubar, wenn der Eingriff von einem erfahrenen Operateur bei guter Bildgebung in einem adäquat ausgestatteten Eingriffsraum mit der Möglichkeit der Komplikationsbehandlung durchgeführt wird.
Hauptrisiko sehen wir in einer unkritischen Ausweitung der Indikationen durch Anwender ohne operative Wirbelsäulenerfahrung.

\section{Korrespondierender Autor Dr. M. Bierschneider}

BG-Unfallklinik Murnau,

Prof.-Küntscher-Straße 8, 82418 Murnau

E-Mail: bierschneider@bgu-murnau.de

Interessenkonflikt: Der korrespondierende Autor versichert, dass keine Verbindungen mit einer Firma, deren Produkt in dem Artikel genannt ist, oder einer Firma, die ein Konkurrenzprodukt vertreibt, bestehen.

\section{Literatur}

1. Aebli N, Krebs J, Davis G et al. (2002) Fat embolism and acute hypotension during vertebroplasty. An experimental study in sheep. Spine27: 460-466

2. Belkoff SM, Mathis JM, Fenton DC et al. (2001) An ex vivo biomechanical evaluation of an inflatable bone tamp used in the treatment of compression fracture. Spine 26:151-156

3. Berlemann U, Ferguson SJ, Nolte LP et al. (2002) Adjacent vertebral failure after vertebroplasty. J Bone Joint Surg Br 84-B: 748-752

4. Bernhard J, Heini PF, Villiger PM (2003) Asymptomatic diffuse pulmonary embolism caused by acrylic cement: an unusual complication of percutaneous vertebroplasty. Ann Rheum Dis 62: 85-86

5. Boszczyk BM, Bierschneider M, Schmid K et al. (2002) Kypholasty - a review of current knowledge and operative techniques. Eur Spine J 11:612

6. Chen HL, Wong CS, Ho ST et al. (2002) A lethal pulmonary embolism during percutaneous vertebroplasty. Anesth Analg 95: 1060-1062
7. Dahl OE, Garvic LJ, Lyberg T (1994) Toxic effects of methylmethaccrylate monomer on leucocytes and endothelial cells in vitro. Acta Orthop Scand 65: 147-153

8. Davis JW, Grove JS, Wasnich RD et al. (1999) Spatial relationships between prevalent and incident spine fractures. Bone 24: 261-264

9. Deramond H, Wright NT, Bellkoff SM (1999) Temperature elevation caused by bone cement polymerization during vertebroplasty. Bone [Suppl 2] 25: $17-21$

10. Finch L, Cheng SG, Steinberg KP et al. (2002) Polymethylmethacrylate pulmonary emboli. Clin Pulmonol Med 9: 133-134

11. Gangi A, Dietemann JL, Guth S et al. (1999) Computed tomography (CT) and fluoroscopy-guided vertebroplasty: results and complications in 187 patients. Semin Intervent Radiol 16: 137-142

12. Garfin SR, Hansen AY, Reiley MA (2001) Kyphoplasty and vertebroplasty for the treatment of painful osteoporotic compression fractures. Spine 26 : 1511-1515

13. Garfin SR, Lin G, Lieberman I et al. (2001) Retrospective analysis of the outcomes of balloon kyphoplasty to treat vertebral body compression fracture refractory to medical management. Eur Spine J 10: S7-S8

14. Harrington KD (2001) Major neurological complications following percutaneous vertebroplasty with polymethylmethacrylate. J Bone Joint Surg Am 84A: 1070-1073

15. Jang JS, Lee SH, Jung SK (2002) Pulmonary embolism of polymethylmethacrylate after percutaneous vertebroplasty. Spine 27: E416-E418

16. Kaufmann TJ, Jensen ME, Ford G et al. (2002) Cardiovascular effects of polymethylmethacrylate use in percutaneous vertebroplasty. Am J Neuroradiol 23: 601-604

17. Lee BJ, Lee SR, Yoo TY (2002) Paraplegia as a complication of percutaneous vertebroplasty with polymethylmethacrylate. Spine 27: E419-E422 
18. Lieberman IH, Dudeney S, Reinhardt MK et al. (2001) Initial outcome and efficacy of ,kyphoplasty ${ }^{\prime \prime}$ in the treatment of painful osteoporotic vertebral compression fractures. Spine 26: 1631-1638

19. Padovani B, Kasriel O, Brunner P et al. (1999) Pulmonary embolism caused by acrylic cement: a rare complication of percutaneous vertebroplasty. Am J Neuroradiol 20: 375-377

20. Piergiogio T, Abdelmounenen $\mathrm{Y}$, Corno AF et al. (2002) Management of pulmonary embolism during acrylic vertebroplasty. Ann Thorac Surg 74: 1706-1708

21. Ratliff J, Nguyen T, Heiss J (2001) Root and spinal cord compression from methylmethacrylate vertebroplasty. Spine 26: E300-E302

22. Ross PD, Genant HK, Davis JW et al. (1993) Predicting vertebral fracture incidence from prevalent fractures and bone density among none-black, osteoporotic women. Osteoporosis Int 3: 120-126

24. Scroop R, Eskridge J, Britz GW (2002) Paradoxical cerebral embolization of cement during intraoperative vertebroplasty: case report. Am J Neuroradiol 23: $868-870$

23. Shapiro S, Abel T, Purvines S (2003) Surgical removal of epidural and intradural polymethylmetacrylate extravasation complicating percutaneous vertebroplasty for an osteoporotic lumbar compression fracture. Case report. J Neurosurg [Suppl] 98: 90-92

25. Tozzi P, Abdelmoumene Y, Corno AF et al. (2002) Management of pulmonary embolism during acrylic vertebroplasty. Ann Thorac Surg 74: 1706-1708

26. Wilson DR, Myers ER, Mathis JM et al. (2000) Effect of augmentation on the mechanics of vertebral wedge fractures. Spine 25: 158-165 